\title{
Automatic Delineation of Sulci and Improved Partial Volume Classification for Accurate 3D Voxel-Based Cortical Thickness Estimation from MR
}

\author{
Oscar Acosta ${ }^{1}$, Pierrick Bourgeat ${ }^{1}$, Jurgen Fripp $^{1}$, Erik Bonner $^{1}$, Sébastien Ourselin $^{2}$, \\ and Olivier Salvado ${ }^{1}$ \\ ${ }^{1}$ The Australian e-Health Research Centre, ICTC-CSIRO, Brisbane, QLD, Australia \\ \{Oscar.Acosta, Pierrick.Bourgeat, Jurgen. Fripp, Erik.Bonner, \\ Olivier.Salvado\} @csiro.au \\ ${ }^{2}$ Centre for Medical Image Computing, University College London, UK \\ S.Ourselin@cs.ucl.ac.uk
}

\begin{abstract}
Accurate cortical thickness estimation in-vivo is important for the study of many neurodegenerative diseases. When using magnetic resonance images (MRI), accuracy may be hampered by artifacts such as partial volume (PV) as the cortex spans only a few voxels. In zones of opposed sulcal banks (tight sulci) the measurement can be even more difficult. The aim of this work is to propose a voxel-based cortical thickness estimation method from MR by integrating a mechanism for correcting sulci delineation after an improved partial volume classification. First, an efficient and accurate framework was developed to enhance partial volume classification with structural information. Then, the correction of sulci delineation is performed after a homotopic thinning of a cost function image. Integrated to our voxel-based cortical thickness estimation pipeline, the overall method showed a better estimate of thickness and a high reproducibility on real data $\left(R^{2}>0.9\right)$. A quantitative analysis on clinical data from an Alzheimer's disease study showed significant differences between normal controls and Alzheimer's disease patients.
\end{abstract}

\section{Introduction}

Automatic measurement of cortical thickness from 3D magnetic resonance (MR) images can aid diagnosis and longitudinal studies of a wide variety of neurodegenerative pathologies, such as Alzheimer's disease. The approaches previously proposed in the literature can be broadly categorised as mesh-based [6] and voxel-based [1|2|3|4|5]. Operating directly on the $3 \mathrm{D}$ voxel grid, voxel based techniques are more computationally efficient but less robust to noise and missegmentation as they typically lack the mechanisms required to assess and correct topological errors in gray matter (GM), white matter (WM) and cerebrospinal fluid (CSF) segmentations. Tissue classification and cortical thickness measurement can be considerably affected by partial volume (PV) effect due to the finite resolution of MRI compared to the size of the considered structures. Thus, a voxel representing more than one tissue type in opposite banks of tight sulci may appear fused as CSF is not detected between the folds of gray matter. This results in erroneously high thickness estimates for these regions.

D. Metaxas et al. (Eds.): MICCAI 2008, Part I, LNCS 5241, pp. 2532612008.

(C) Springer-Verlag Berlin Heidelberg 2008 
Few approaches have been proposed to estimate and cut misdetected sulci. In [2], the GM/CSF partial volume voxels between opposing banks of sulci are identified using a combination of their distance to the white matter and the classification of neighbouring voxels. This method, however, requires removal of noise and intensity inhomogeneities in a prior step. Hutton [1] uses the thickness to identify deep sulci from GM layers growing consecutively from the WM. However, no additional information is used to ensure that sulci are correctly identified and does not account for partial volume information to accurately compute the thickness. Diep [4] proposed to cut sulci using the probability of the voxel being CSF in abnormally thick areas, but it lacks a partial volume model to properly identify CSF voxels buried inside deep sulci.

Unlike these approaches, we propose a technique based on an improved partial volume (PV) classification and topological operators to better delineate the buried sulci and to accurately compute the thickness. Firstly, the proposed partial volume classification method accounts for the distance to the WM, favoring the detection of GM/CSF voxels in oposed sulcal banks. Secondly, for detecting the remaining non-classified mixed voxels in buried sulci (100\% GM) we used a distance ordered homotopic thinning DOHT [16] of a sulcal function, controlled by a surface terminality criteria. Then, the resulting skeletonized surface combined with the partial volume maps are used to correct the hard segmentations for computing the thickness. After correction of WM, GM and CSF segmentations, the GM thickness is computed using the Laplacian definition [7] with a more efficient PDE implementation [8], and using the PV maps for initialisation [5] achieving subvoxel accuracy.

\section{Method}

\subsection{Pure Tissue Segmentation}

Based on the expectation maximisation segmentation (EMS) algorithm [9], we have implemented a method for segmentation of brain tissues into GM, WM and CSF which includes a polynomial based bias field correction and Markov random fields to reduce the effects of noise. The Colin atlas and associated priors are first affinely registered to the data using a robust block matching approach [10], followed by a diffeomorphic Demons non-rigid registration [11]. The registered priors are then used to initialise the EMS and enforce spatial consistency throughout the segmentation. Finally, hard segmentations are obtained after the EMS by labelling each voxel with the most probable tissue.

\subsection{Partial Volume Estimation}

PV along the interfaces of pure tissues is estimated by modeling the mixture of pure tissues and performing a maximum a posteriori classification (MAP). We improved the two-stage procedure relying on both intensity and spatial information used previously by [13 14 15]. The Euclidean distance from the WM (and its gradient) are combined with the spatial information to favour classification of GM/CSF mixed voxels in the GM, improving the delineation of the sulci. Three pure classes (GM, WM, CSF) 
and two mixture classes (GM/CSF, GM/WM) are considered. Thus, the labels are restricted to the set $\Gamma=\{\mathrm{GM}, \mathrm{CSF}, \mathrm{WM}, \mathrm{CSF} / \mathrm{GM}, \mathrm{GM} / \mathrm{WM}\}$. Since voxels containing $\mathrm{PV}$ are mostly present along boundaries, we assume that each voxel contains at most two tissues [12], and PV classification is restricted to the region formed by a dilated GM (radius 4). As in [14], labels $j / k$ indicate mixed voxels of the pure tissue types $j$ and $k$. The output of the classification is an image $C$ of labels for each voxel as $C=\left\{c_{i}: i=1, . ., N, c_{i} \in \Gamma\right\}$.

Intensity model. Pure voxels are assumed to have a Gaussian probability density function, defined by its mean $(\mu)$ and standard deviation $(\sigma)$, whereas mixed voxels are modeled with a probability density function as in [12]:

$$
p\left(x_{i} \mid c_{i}=j / k\right)=\int_{0}^{1} \frac{1}{\sqrt{2 \pi \operatorname{det}(\Sigma(w))}} \exp \left[-\frac{1}{2}\left(x_{i}-\mu(w)\right)^{T} \Sigma^{-1}\left(x_{i}-\mu(w)\right)\right] d w
$$

where the mean and covariance matrix of the mixture are a function of the means and covariances of the pure tissue classes as $\mu(w)=w \mu_{j}+(1-w) \mu_{k} ; \Sigma(w)=w^{2} \Sigma_{j}+$ $(1-w)^{2} \Sigma_{k}$ and $w$ represents the fraction of $j$ within the mixed voxel.

Local spatial interaction model. To take into account dependency on the neighbouring tissue types, a Markov prior that models local spatial interaction was implemented. The labelling is performed using a Potts model. As in ([13 14 15]), we use the ICM algorithm [17] to search for the optimal labelled image as

$$
\lambda_{i}^{n+1}=\arg \max _{\gamma \in \Gamma}\left[\log p\left(x_{i} \mid \gamma\right)-2 \beta \sum_{q \in N_{q}} \frac{\delta\left(\lambda_{q}^{n}, \gamma\right)}{d(i, q)}\right]
$$

where $d(i, q)$ is the distance between centers of voxels $i$ and $q, \lambda_{i}^{n}$ is the label at the $n$-th update, $\beta$ controls the strength of the prior (typically $\beta=0.05$ ) and $N_{q}$ represents the set of voxels in the 26-neighbourhood of $i$. Building on [13 14] who used a similar approach, we modified $\delta\left(\lambda_{i}, \lambda_{q}\right), \lambda_{i}$ and $\lambda_{q}$ being the labels of the voxels $i$ and $q$. We introduced two functions, namely $f_{1}$ and $f_{2}$ that modulate the influence of the Euclidean distance to the WM, $D_{W M}$. The design of $f_{1,2}$ takes advantage of local maxima where $\left\|\nabla D_{W M}\right\| \rightarrow 0$ when a voxel likely belongs to a sulci (Fig 1 (c)). Thus,

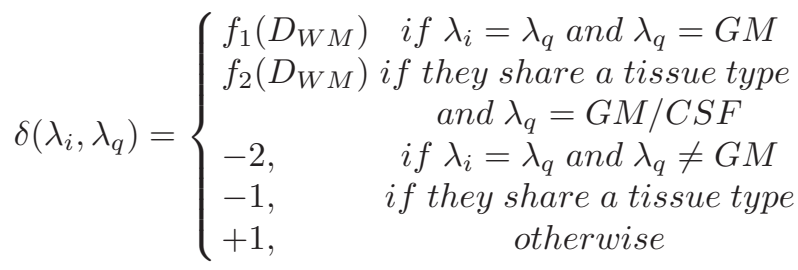

where

$$
f_{1,2}\left(D_{W M}\right)=A \cdot \exp \left[-\alpha D_{W M}\right] \cdot \exp \left[-k\left\|\nabla D_{W M}\right\|^{2}\right]+B
$$

For $f_{1}: A=-1, \alpha=0.1, B=-1, k=1$ and for $f_{2}: A=1, \alpha=0.1, B=-2, k=1$. $f_{1}$ varies between $[-1,-2]$, and tends to attenuate the influence of neighbouring GM voxels when $\left\|\nabla D_{W M}\right\|$ goes to zero. Conversely, $f_{2}$ varies between $[-2,-1]$ tending to reinforce the influence of labels GM/CSF in the neighbourhood when $\left\|\nabla D_{W M}\right\|$ 
tends to zero. Thus, these functions favor both reclassification of pure tissue voxels GM as GM/CSF (by $f_{1}$ ) and preservation of classified voxels GM/CSF (by $f_{2}$ ). The design of $f_{1,2}$ follows the same reasoning as for detecting plate-like structures [18]. As depicted in Fig. 1(d) the sulci are well delineated in the resulting GM partial volume maps (GMPVC).

Fractional content. Once voxels have been classified as a mixture of pure tissue, their fractional content $F_{j / k}$ between tissue $j$ and $k$ is computed as in [12] using the bias corrected intensity $\bar{x}_{i}$, and the means of the two pure tissue types $\mu_{j}$ and $\mu_{k}$, such that:

$$
F_{j / k}=U\left(\frac{\mu_{j}-\bar{x}_{i}}{\mu_{j}-\mu_{k}}\right)
$$

where $U(\cdot)$ is a limiter restricting the range of the fractional content to $[0,1]$. The partial volume coefficients (PVC) map of GM (and similarly for WM and CSF), is defined as $\mathrm{GMPVC}=F_{\mathrm{GM} / \mathrm{WM}} \cup F_{\mathrm{GM} / \mathrm{CSF}}$.

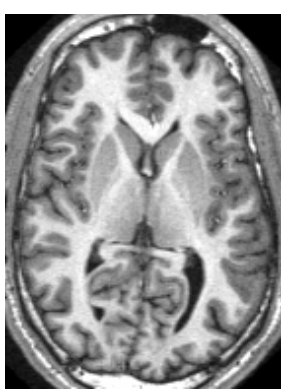

(a) T1W-MRI

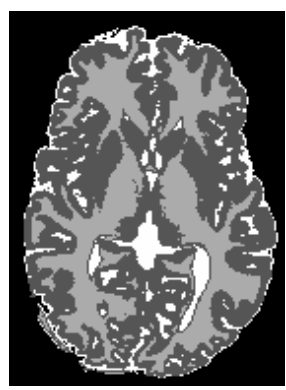

(b) EMS segmentation

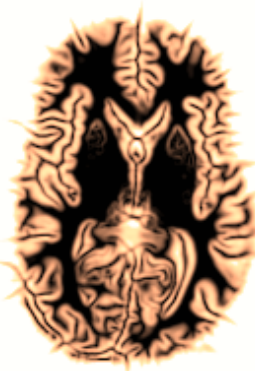

(c) $\left\|\nabla D_{W M}\right\|$

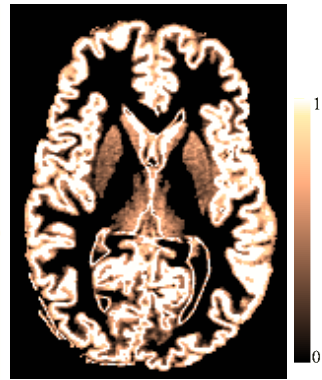

(d) GMPVC map

Fig. 1. (a) Axial cut plane of a T1-W MR Image, (b) pure tissue segmentation, (c) gradient of the Euclidean distance to the WM (\| $\left.\nabla D_{W M} \|\right)$, (d) GM partial volume image (GMPVC map)

\subsection{Sulci Delineation and Correction of Hard Segmentation}

To complete the delineation of sulci in very tight zones not detected after the partial volume classification (Fig. 2 $\mathrm{d}$ ), a procedure based on homotopic thinning is employed. Thus, the hard segmentations are corrected by the medial surface (3D skeleton) from the local minima of a sulci likelihood function, $F_{s}$, computed from the WM and CSF partial volume maps (WMPVC,CSFPVC) as

$$
F_{s}=\left(1-C S F P V C_{\sigma}\right)\left\|\nabla D_{W M P V C_{50}}\right\|
$$

where $D_{W M P V C_{50}}$ is the distance to the WMPVC (PVC>50\%) and CSFPVC $\sigma$ is a Gaussian convolved CSFPVC map $(\sigma=1)$. The CSFPVC shifts the local minima of $F_{s}$ towards the maximum of CSF, overcoming the problem of sulci asymmetry. The thinning of $F_{s}$ is performed using a two stage distance-ordered homotopic thinning (DOHT) [16]. However, rather than only using the center of maximal balls technique in the first stage, which makes the method scale-dependent, the first skeleton is completed by adding all the local minima over $F_{s}$ following the direction of $\nabla D_{W M P V C_{50}}$. In the second step, voxels are recursively deleted until the medial surface is obtained. 


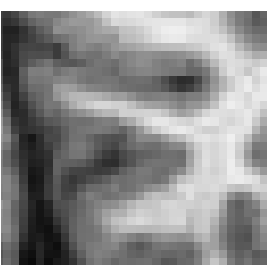

(a) T1W-MRI

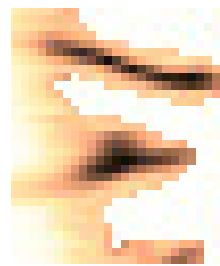

(e) $F_{s}$

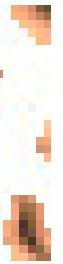

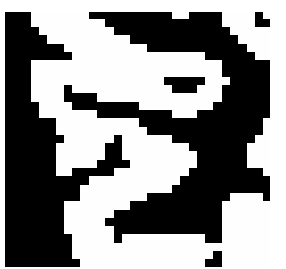

(b) GM segmentation

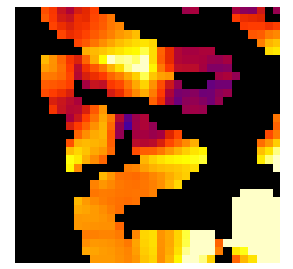

(f) Thickness w/o PVC

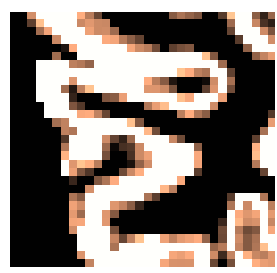

(c) GMPVC M1

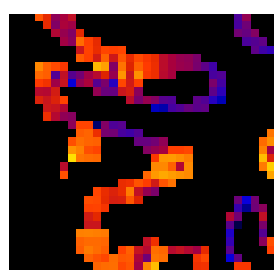

(g) Thickness PVC M1

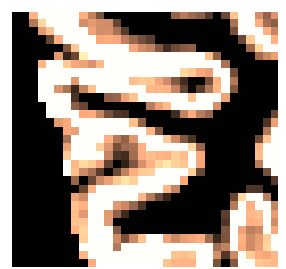

(d) GMPVC M2

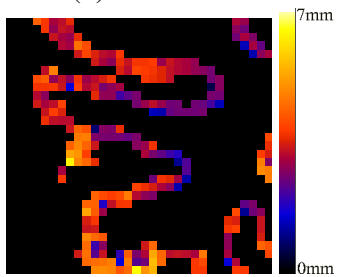

(h) Corrected thickness

Fig. 2. (a) Deep sulci in original T1W-MRI, (b) GM hard segmentation (No PVC), (c) GMPVC map with method (M1) [13] and (d) with the proposed method (M2), (e) cost function $F_{s}$ where the DOHT is performed using [16], (f-h) corresponding thickness estimation: (f) in the GM hard segmentation, without taking into account the PVC, (g) using GMPVC M1 and (h) using improved GMPVC map after correcting sulci delineation

\subsection{Cortical Thickness Estimation}

Cortical thickness is computed using Jones's approach [7] where Laplace's equation is solved in the GM volume (with the WM and CSF voxels adjacent to the boundaries of the GM set to fixed potentials) such that $\nabla^{2} f(\mathbf{x})=0$.

The solution $f(\mathbf{x})$ is a scalar field which divides the cortex into a set of equipotential sublayers. The normalised gradient of the Laplace solution provides streamlines between the WM and CSF, which do not intersect, are locally perpendicular to the equipotential sublayers, and provide a unique correspondence between the two boundaries. The tangent vectors of the correspondence trajectories $\vec{T}$ correspond to the normalised gradient vector field of $f(\mathbf{x})$, regularised using a Gaussian function $G_{\sigma}$ with $\sigma=1$ such that $\vec{T}=G_{\sigma} * \frac{\nabla f}{\|\nabla f\|}$.

Whereas Jones [7] explicitly traces streamlines (Lagrangian approach), Yezzi [8] proposed a more efficient method which involves solving a pair of first order linear partial differential equations (PDE) without any explicit construction of correspondences (Eulerian approach). We implemented a hybrid Eulerian-Lagrangian approach [5], in which the PV information is used to initialise the PDE, achieving subvoxel accuracy. The PV content of each boundary voxel is combined with the direction of the tangent field, to accurately measure the position of the boundary through ray-casting. The implementation is based on a dichotomy search, with decreasing stepsize down to $\epsilon=1 / 10^{-3}$ of the voxel size. In opposed sulcal banks the position of the boundary is computed as the point where the GMPVC is minimum. 


\section{Experiments and Results}

\subsection{Partial Volume Classification}

To evaluate the partial volume classification, we used the simulated T1W MR 3D images $\left(1 \mathrm{~mm}^{3}\right.$ voxels) from BrainWeb [19], with noise levels ranging from $1 \%$ to $9 \%$. The ground truth was the simulated partial volume image with $0 \%$ noise. We compared the proposed partial volume classification approach $\left(f\left(D_{W M}\right)\right)$ against other existing methods [13] using the root mean squared error (RMS). The means for pure tissue were derived either from the EMS segmentation or recomputed over the segmented voxels from the EMS hard segmentation (M1). Fig. 3 (a) depicts the results. Compared to the other methods, the RMS was considerably reduced. On average $14 \%$ compared to M1, with the greatest differences in the GM (12.2\%) and CSF (29.3\%) as expected.

\subsection{Deep Sulci Cutting and Cortical Thickness Estimation on Real Data}

From the OASIS database [20], we extracted 20 young healthy subjects who underwent 4 scans at baseline and 4 more scans during a subsequent session after a short delay (less than 90 days). For each session, an average motion-corrected image (co-registered average of all available data) was used for our reliability test. The scans were T1W MPRAGE with isotropic $1 \mathrm{~mm}^{3}$ resolution. By using the proposed method, we found a mean \pm (std. dev.) cortical thickness over the whole brain of $2.49 \mathrm{~mm} \pm 0.11$ for all the subjects, which is within the accepted range of cortical thickness for healthy young adults (Fig 3b). When the PV is not taken into account as in [8], the computed mean thickness was $4.69 \mathrm{~mm} \pm 0.11$.

The sum of square of differences was computed for each ROI of the AAL template to assess the error between the two measurements (Fig.4). The cerebellum and subcortical

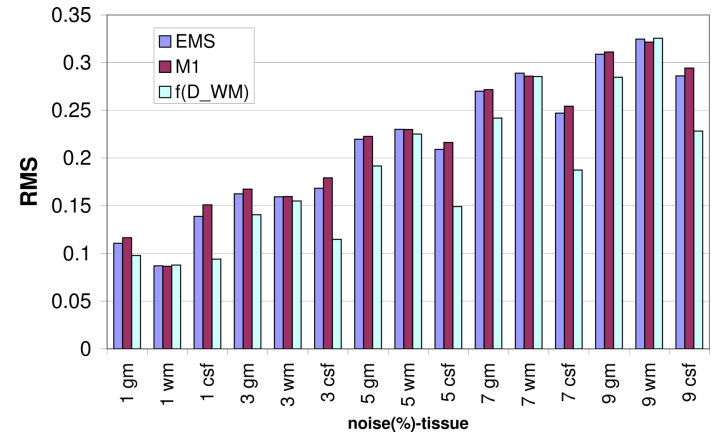

(a) RMS partial volume

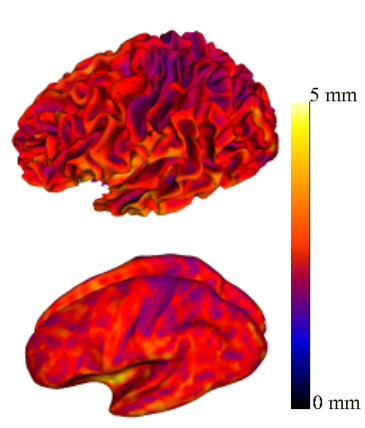

(b) Rendering of a thickness map.

Fig. 3. (a) RMS comparisons between different methods for computing partial volume: i) Using the means from the EMS, ii) computing the means from the hard segmentation (M1) and iii) including the distance functions $f_{1,2}\left(D_{W M}\right)$ as proposed. (b) Top: Rendering of a thickness map for a healthy young adult shown over the WM/GM surface and Bottom: over a partially inflated surface. 


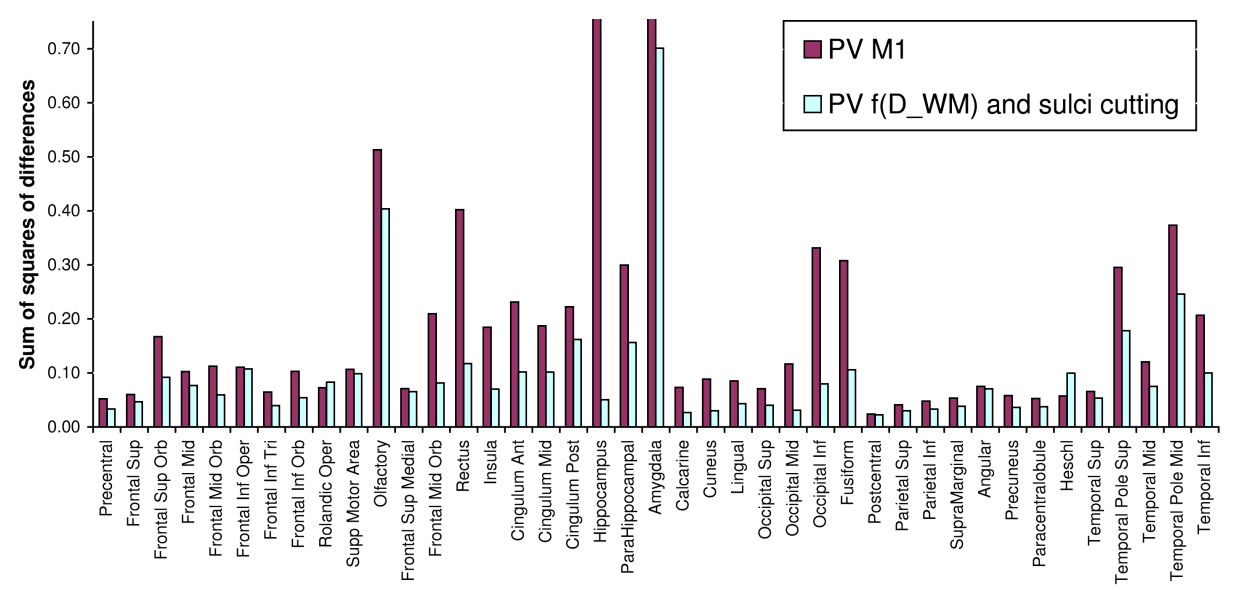

Fig. 4. Sum of square of differences of regional cortical thickness between baseline and repeat for 20 young healthy subjects

nuclei were excluded from the analysis. We found a significant reduction of error in the temporal lobe, with a drastic reduction in the hippocampal region when comparing the proposed method (Improved PVC and cut sulci) with the measure performed after partial volume classification using [13] (PVM1). The Pearson coefficient for all the regions was above 0.9 , except for the olfactory $(0.79)$ and a paired t-test did not reveal any significant differences between the 2 measurements $(p>0.01)$.

The technique was also applied to a database of 3D MPRAGE MRI from our Alzheimer's disease (AD) study (The Australian Biomarker, Imaging And Lifestyle Study-AIBL). We extracted 23 subjects: 12 healthy elderly (HE) and 11 AD patients. The mean age was $70.4 \pm 7.35$ for the $\mathrm{HE}$ and $69.5 \pm 6.7$ for the AD. Regional analysis showed significant differences $(\mathrm{p}<0.001)$ in the hippocampus $(8.5 \%$ atrophy), parahippocampus $(6.6 \%)$ and posterior cingulum $(5 \%)$ and $(\mathrm{p}<0.01)$ in the temporal and frontal lobes between $\mathrm{HE}$ and $\mathrm{AD}$. The p-values were greater when using M1 for almost all the regions indicating that the statistical significance increased using the proposed approach.

\section{Discussion and Conclusions}

In this work we have presented a novel voxel-based method for accurate cortical thickness estimation, which integrates a mechanism for delineating deep sulci based on partial volume classification. The main contribution lies in two points: Firstly, it introduces an improved partial volume classification method which favours labelling of mixed $\mathrm{GM} / \mathrm{CSF}$ voxels. Secondly, it corrects hard segmentations (GM voxels that must be reclassified as GM/CSF) after a homotopic thinning of a sulcal function, obtained from the resulting PVC maps. Partial volume classification was improved when compared 
with other methods using the BrainWeb fuzzy maps as ground truth. Both fractional tissue content estimation and thinning resulted in reliable brain tissue segmentation for more accurate cortical thickness estimation. Our method showed a high reproducibility on real data, with an extremely good agreement between the baseline and repeat scan. Furthermore, a preliminary quantitative analysis on clinical data showed significant differences between healthy elderly and Alzheimer's disease patients, consistent with results previously published in the literature. In the future, we plan to use our technique on clinical data to study cortical atrophy in Alzheimer's disease and other neurodegenerative diseases. We intend also to develop voxel-based techniques for intersubject comparisons, a challenging issue given the large anatomical variability between patients.

\section{References}

1. Hutton, C., De Vita, E., et al.: Voxel-based cortical thickness measurements in MRI. Neuroimage (2008)

2. Lohmann, G., Preul, C., Hund-Georgiadis, M.: Morphology-based cortical thickness estimation. In: Taylor, C.J., Noble, J.A. (eds.) IPMI 2003. LNCS, vol. 2732, pp. 89-100. Springer, Heidelberg (2003)

3. Srivastava, S., Maes, F., et al.: An automated 3D algorithm for neo-cortical thickness measurement. In: Ellis, R.E., Peters, T.M. (eds.) MICCAI 2003. LNCS, vol. 2879, pp. 488-495. Springer, Heidelberg (2003)

4. Diep, T.M., Bourgeat, P., Ourselin, S.: Efficient use of cerebral cortical thickness to correct brain MR segmentation. In: IEEE-ISBI 2007, Washington DC, USA, pp. 592-595. IEEE, Los Alamitos (2007)

5. Bourgeat, P., Acosta, O., et al.: Improved cortical thickness measurement from MR images using partial volume estimation. In: IEEE-ISBI 2008, Paris, France, pp. 205-208. IEEE, Los Alamitos (2008)

6. Lee, J., et al.: A novel quantitative cross-validation of different cortical surface reconstruction algorithms using MRI phantom. Neuroimage 31(2), 572-584 (2006)

7. Jones, S., Buckbinder, B., Aharon, I.: Three-dimensional mapping of cortical thickness using Laplace's equation. HBM 11(1), 12-32 (2000)

8. Yezzi, A., Prince, J.: An Eulerian PDE approach for computing tissue thickness. IEEETMI 22(10), 1332-1339 (2003)

9. van Leemput, K., Maes, F., et al.: Automated model-based bias field correction of MR images of the brain. IEEE-TMI 18(10), 885-896 (1999)

10. Ourselin, S., Roche, A., et al.: Reconstructing a 3D structure from serial histological sections. IVC 19(1), 25-31 (2001)

11. Vercauteren, T., Pennec, X., et al.: Non-parametric diffeomorphic image resgistration with the demons algorithm. In: Ayache, N., Ourselin, S., Maeder, A. (eds.) MICCAI 2007, Part II. LNCS, vol. 4792, pp. 319-326. Springer, Heidelberg (2007)

12. Santago, P., Gage, H.: Quantification of MR brain images by mixture density and partial volume modeling. IEEE-TMI 12(3), 566-574 (1993)

13. Shattuck, D., Sandor-Leahy, S., et al.: Magnetic resonance image tissue classification using a partial volume model. Neuroimage 13(5), 856-876 (2001)

14. Tohka, J., Zijdenbos, A., Evans, A.: Fast and robust parameter estimation for statistical partial volume models in brain MRI. NeuroImage 23(1), 84-97 (2004) 
15. Kim, J., Singh, V., et al.: Automated 3D extraction and evaluation of the inner and outer cortical surfaces using a Laplacian map and partial volume effect classification. NeuroImage 27(1), 210-221 (2005)

16. Pudney, C.: Distance-Ordered Homotopic Thinning: A Skeletonization Algorithm for 3D Digital Images. CVIU 72(3), 404-413 (1998)

17. Besag, J.: On the statistical analysis of dirty pictures. J. Roy. Stat. Soc. 48, 259-302 (1986)

18. Frangi, A., Niessen, W., et al.: Multiscale Vessel Enhancement Filtering. In: Wells, W.M., Colchester, A.C.F., Delp, S.L. (eds.) MICCAI 1998. LNCS, vol. 1496, pp. 130-138. Springer, Heidelberg (1998)

19. Cocosco, C., Kollokian, V., et al.: Brainweb: Online interface to a 3D MRI simulated brain database. NeuroImage (Proc. of 3-rd Int. Conf. Func. Mapp Human Brain) 5, S425 (1997)

20. Marcus, D.S., Wang, T.H., et al.: Open access series of imaging studies (OASIS): Crosssectional MRI data in young, middle aged, nondemented, and demented older adults. J. Cogn. Neurosci. 19, 1498-1507 (2007) 